\title{
An Unusual Case of Esophageal Rupture
}

\author{
Nizar Tejani ${ }^{1}$, Sylvia Bowditch ${ }^{1}$, Kai Engstad ${ }^{2}$ and Leonard Ranasinghe ${ }^{1^{\star}}$ \\ ${ }^{1}$ California Northstate University College of Medicine, California, USA \\ ${ }^{2}$ Legacy Emanuel Medical Center, Portland, Oregon, USA
}

${ }^{*}$ Corresponding author: Leonard Ranasinghe, Department of Emergency Medicine, California Northstate University College of Medicine, California, USA, Tel: +916 686-7929; E-mail: Leonard.Ranasinghe@cnsu.edu

Received: 15 April, 2017; Accepted: 25 May, 2018; Published: 05 June, 2018

\begin{abstract}
Boerhaave Syndrome is a rare entity which occurs as a result of rupture of the distal esophagus leading to a high rate of morbidity and mortality. It occurs most frequently in patients with bulimia or alcoholism because of the increased episodes of emesis and underlying esophageal fragility. We present a unique case of a 38-year old firefighter / paramedic with no prior risk factors for esophageal rupture who sustained this injury after three bouts of emesis. The diagnosis of Boerhaave Syndrome was initially missed by the emergency physician, who attributed the patient's condition to nephrolithiasis. A radiologist's attention to detail ultimately led to the correct diagnosis. Our patient underwent surgery to repair the tear and required extensive follow-up care. Pathophysiology, epidemiology, imaging, management, and complications are discussed in this case report.
\end{abstract}

\section{Keywords}

Boerhaave Syndrome; Case Report; Emergency Medicine; Esophageal Rupture; Esophageal Tear; Gastroenterology

\section{Introduction}

Boerhaave Syndrome is characterized by transmural rupture of the distal esophagus. It was first coined by Dr. Herman Boerhaave in 1724, who described the death of nobleman Baron Jan von Wassenaer. Wassenaer died after a bout of excessive drinking and consumption of food. While performing the autopsy, Boerhaave discovered a tear in the distal esophagus with spilled food particles in the chest cavity causing mediastinal emphysema [1]. In accordance with this original case, Boerhaave Syndrome continues to be associated with an overindulgence of food and alcohol and is therefore most frequently seen in individuals with bulimia and alcoholism [2]. Boerhaave Syndrome is thought to be due to failure of the cricopharyngeus muscle to relax during emesis, which leads to a pathologic increase in esophageal intraluminal pressure and subsequent rupture.

Spontaneous esophageal ruptures are extremely uncommon, with fewer than 150 cases reported per year in the US $[3,4]$. Boerhaave Syndrome is a frequently overlooked diagnosis due to its rareness and nonspecific symptoms. Early recognition and intervention are critical for patient survival. It is the most lethal gastrointestinal perforation, with a mortality rate of nearly $100 \%$ if left untreated for more than 48 hours [5].

\section{Case Presentation}

A 38-year old firefighter / paramedic felt nauseated after lunch and vomited three times. He then experienced an excruciating pain in his left flank and upper abdomen and was immediately taken to the nearest Emergency Department (ED). The patient was in excellent health prior to this incident; his past history showed only seasonal rhinitis. His sole medication was a steroid nasal spray. On arrival to the ED his vital signs were: BP: 100/80, P: 140, RR: 16. Suspecting a possible left-sided kidney stone, the ED physician prescribed intravenous narcotics and ordered a CT of the abdomen and pelvis without contrast. Although the patient was a narcotic-naïve individual, his pain was not completely resolved with the medication he received.

The radiologist reported that the CT of the abdomen and pelvis did not show any kidney stones, but there was evidence of pneumomediastinum. A subsequent chest CT with contrast showed an approximately $6 \mathrm{~cm}$ esophageal tear (Figure 1). An esophagogram (Figure 2) confirmed the distal esophageal tear. That night, the patient was brought to the operating room, where he underwent a left thoracotomy and primary two-layer repair with a 
pedicled intercostal muscle flap. The tear was found to be located 3-4 cm above the GE junction. The chest was grossly contaminated with large amounts of food and the surrounding tissues were inflamed. After three weeks of close monitoring, the patient was deemed stable for discharge. At this time, he had a small right pleural effusion. About ten days after discharge, the patient's father noticed that he appeared ill, diaphoretic, and tachycardic. The patient was immediately taken to a nearby ED and found to be septic secondary to a right-sided empyema. Chest tubes were placed for drainage, and the patient was admitted to the ICU. A few days later, a chest CT and another esophagogram revealed a leak at the previously-repaired distal esophageal tear. An esophageal stent was placed under endoscopy by a gastroenterologist to facilitate healing of the tear. The empyema improved significantly, but the patient continued to have tachycardia. A repeat chest CT showed resolution of the empyema. However, it also showed two new developments: a markedly elevated left hemidiaphragm and a pericardial effusion. The latter was confirmed by an echocardiogram. A pericardial window procedure was done by a cardiothoracic surgeon and $700 \mathrm{ml}$ of pericardial fluid was removed. Culture of the fluid was negative and the patient recovered from the effusion without any complications. A few weeks later a follow-up CT indicated that his esophageal stent had migrated into the stomach. A second endoscopy was done to remove the migrated stent and it was replaced with a longer one. After nearly one month in the hospital, he was discharged. He had close follow-ups with his primary care physician, pulmonologist, gastroenterologist, and cardiothoracic surgeon. Six weeks after discharge, the second esophageal stent was removed and he continued to improve. At this point he had lost forty pounds. During a follow-up chest fluoroscopy, which included a "sniff test," it was noted that the patient's elevated left hemidiaphragm still persisted. The pulmonologist attributed this persistent elevated hemidiaphragm finding to either phrenic nerve neuropraxia or paralysis. The patient was determined to return to work and began his recovery training with short walks, which he gradually increased to jogging. His stamina improved significantly during this period. He successfully completed a physical examination which allowed him to return to work full-time work as a paramedic / firefighter. Nevertheless, the patient's left hemidiaphragm persisted seven months after his esophageal tear (Figure 3).

\section{Presentation/Signs and Symptoms}

Typically Boerhaave's patients will have a history of nausea and vomiting followed by dyspnea and sudden, severe epigastric pain that may radiate to the back or left shoulder. In most cases, air enters the mediastinum and leads to subcutaneous emphysema, which may present as crepitus in the neck [6]. Patients may also exhibit Hammon's sign on auscultation, a pericardial crunching sound that reflects the heart beating against air trapped in the mediastinum. Chest pain, severe vomiting, and subcutaneous emphysema are collectively referred to as Mackler's Triad, a diagnostic hallmark of Boerhaave Syndrome [7].

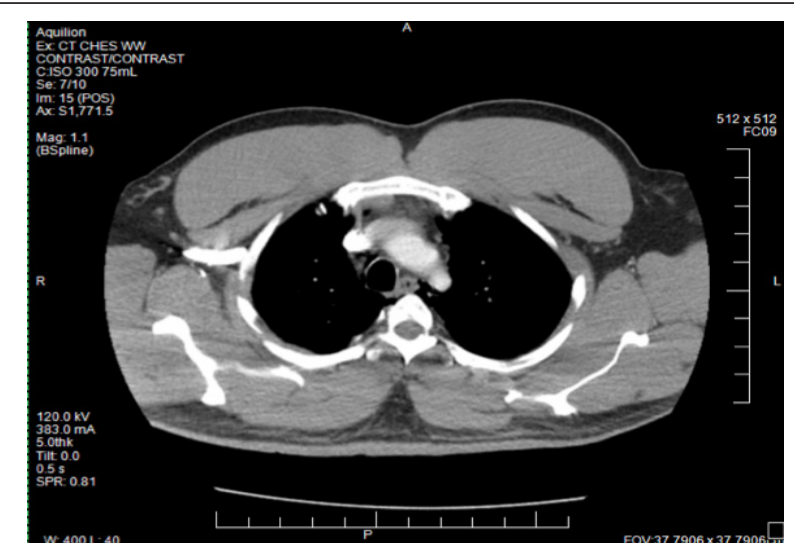

Figure 1: A CT of the chest shows leakage of contents into the mediastinum from a ruptured esophagus. This is highly suggestive of Boerhaave Syndrome and warrants a confirmatory esophagogram.

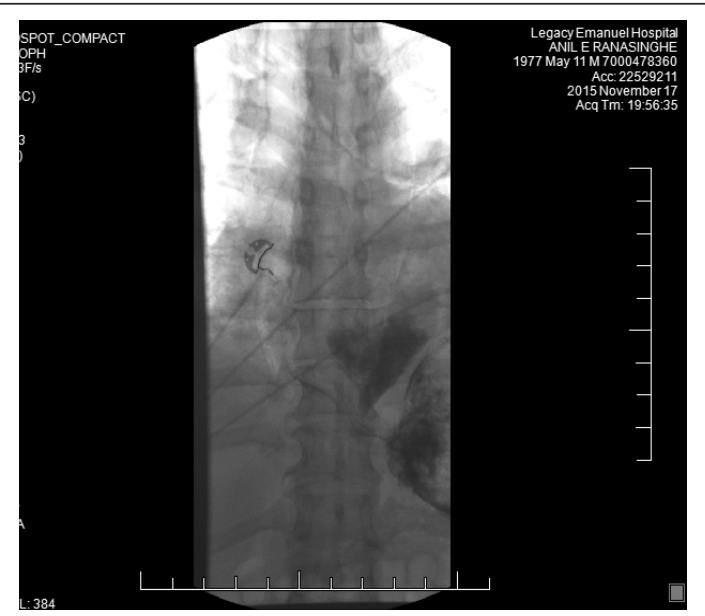

Figure 2: An esophagogram that shows extravasation of contrast, confirming the diagnosis of Boerhaave Syndrome.

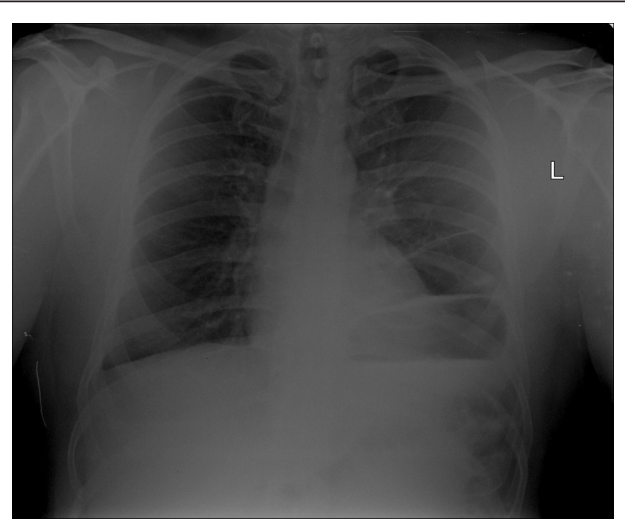

Figure 3: An elevated L diaphragm can be seen on this chest xray. 


\section{Differential Diagnoses}

There are a number of differential diagnoses based on the nonspecific presentation of a patient with Boerhaave Syndrome. The chest pain and dyspnea may mimic cardiorespiratory etiologies, including myocardial infarction, pericarditis, spontaneous pneumothorax, pulmonary embolism, or pneumonia. Detection of amylase from spilled mediastinal contents may arouse suspicion of acute pancreatitis [1]. Based on its gastrointestinal symptoms, Boerhaave Syndrome is most commonly misdiagnosed as a perforated ulcer [8].

\section{Imaging}

The constellation of symptoms in a patient with Boerhaave Syndrome warrants a chest radiograph. Abnormalities on imaging will depend on three critical variables: time between rupture and imaging, location of rupture, and mediastinal-pleural integrity [9]. Chest radiographs of esophageal rupture will commonly exhibit a left-sided pleural effusion with atelectasis [7]. This can be attributed to the relative lack of anatomical support to the left posterolateral aspect of the distal one-third of the esophagus. A pneumomediastinum may also be present on film showing the characteristic, albeit, nonspecific, V sign of Naclerio [10]. This radiographic sign describes subtle mediastinal widening caused by collections of air between the fascial planes of the mediastinum and pleura behind the heart [1]. Water-soluble contrast studies and computerized tomography are used to localize a rupture and to confirm diagnosis of full thickness tear [7].

\section{Management}

Primary management of any patient with Boerhaave Syndrome includes fluid resuscitation, initiation of empiric antibiotics, and placement of nasogastric tube [1]. Aseptic patients with minor ruptures and lack of mediastinitis are candidates for conservative medical management using endoscopic stents [11]. However, these stents must be placed early in order reduce the risk of stricture development and ischemia of the esophageal tissue [11]. Migration of stents is variable but not uncommon and can occur in stents with diameters of less than $28 \mathrm{~cm}[12,13]$. In more complicated cases, the primary determinant of treatment choice and outcome is time after perforation [7]. In patients who present within 24 hours of rupture, emergency transthoracic primary repair remains the gold standard [14]. This procedure involves an approximation of healthy mucosal and submucosal tissue with a reported leak rate of $20 \%$. Patients who present after 24 hours of rupture undergo reinforced primary repair with autologous tissue. In severe cases, the surgical treatment of choice is esophagectomy with delayed reconstruction using colon or supercharged jejunum [7]. Early surgical intervention is strongly recommended to reduce the risk of mortality. However, there are numerous case reports showing successful recovery with nonsurgical management [15]. Healing is monitored through water-soluble contrast imaging [7].

\section{Conclusion}

This patient's diagnosis of Boerhaave Syndrome was a challenging one to arrive upon for several reasons. It is unlikely to be included on a differential diagnosis because of its low incidence. In addition, our patient lacked the typical risk factors for Boerhaave Syndrome because he had no prior history of bulimia or alcoholism. However, Boerhaave Syndrome has been reported to be caused by straining, childbirth, weight lifting, coughing, blunt trauma, seizures, asthma, forceful swallowing, and even just a single bout of emesis [10]. It is prudent to consider Boerhaave Syndrome even in absence of obvious risk factors. Our patient's presentation was typical of Boerhaave Syndrome: severe epigastric and back pain following postprandial emesis. It is likely that the patient's flank pain led to the emergency physician's suspicion of nephrolithiasis rather than an upper GI pathology. Had the radiologist not recommended a chest CT, treatment would have been further delayed and risk of mortality would have increased.

Our patient experienced numerous complications that delayed his recovery, some of which may have been prevented. Sepsis, empyema, leakage of surgical site, and stent migration were consistent with other reported complications of Boerhaave Syndrome $[1,11,16]$. In retrospect, our patient's discharge may have been premature, as he had a small right pleural effusion on discharge and subsequently developed empyema and septicemia. Given the degree of pneumomediastinum, bacterial seeding into the right pleural space may be a likely cause.

Although the esophageal leak was discovered after the appearance of these symptoms, it was later attributed to be their cause. This 'cause and effect' has been described in numerous case reports $[1,16]$. The endoscopic placement of this patient's stent differs from most reported cases. The literature describes the use of stenting as a conservative management technique in early onset. Instead, our patient's stent ( $23 \mathrm{~mm} \times 100 \mathrm{~mm}$ ) was placed following the leak and subsequent empyema and septicemia. He required a second stent placement (23 mm x 150mm) as his original stent had migrated into his stomach nine days after its insertion. This longer stent did not migrate and was removed without complications more than a month later. Our patient's case demonstrates that the length of stent may be a critical variable in prevention of migration. This is not entirely supported by the literature. One particular study comparing stent placements in the treatment of esophageal perforations and ruptures suggested that stent diameter rather than length may be important in the prevention of migration. Based on their limited sample size, Johnsson et al., noted that $28 \mathrm{~mm}$ may be an optimal diameter [13]. Investigation into comparing the efficacy of stent length and diameter may be a promising source of further research. Our patient experienced other complications, including the pericardial effusion and left hemidiaphragm, which are atypical in reported cases [17]. The etiology of these conditions in this case is unclear. The pericardial effusion was treated without further complications. In contrast, the left hemidiaphragm could not be treated as easily and proved to be the greatest barrier to recovery for our patient. 
The early diagnosis of Boerhaave Syndrome is paramount and can be aided with a high index of suspicion. Contrast esophagograms remain a crucial tool in diagnosing the rupture and guiding the treatment options. The extravasation of contrast in our patient's esophagogram mandated urgent surgery. Even if the diagnosis is caught early, patients can still develop numerous complications. Boerhaave patients should be treated in centers that have experience in the management of esophageal injuries and access to the full breadth of surgical, endoscopic, and radiologic treatment.

\section{References}

1. Janjua KJ (1997) Boerhaave's Syndrome. Eponyms in Medicine Revisited. Postgrad Med F 73: 265-270.

2. Hecser L, Siklodi PK, Csiki G, Lungu MF, Jung H, et al. (2011) Boerhaave Syndrome. A Case Report. Rom J Leg Med 19: 283-286.

3. Jagminas L, Silverman RA (1996) Boerhaave's syndrome presenting with abdominal pain and right hydropneumothorax. Am J Emerg Med 14: 53-56.

4. Vidarsdottir H, Blondal S, Alfredsson H, Geirsson A, Gudbjartsson T (2010) Oesophageal perforations in Iceland: a whole population study on incidence, aetiology and surgical outcome. Thorac Cardiovasc Surg 58: 476-480.

5. Brinster CJ, Singhal S, Lee L, Marshall MB, Kaiser LR, et al. (2004) Evolving options in the management of esophageal perforation. Ann Thorac Surg 77: 1475-1483.

6. Vaidya S, Prabhudessai S, Jhawar N, Patankar RV (2010) Boerhaave's Syndrome: Thoracolaparoscopic Approach. J Minim Access Surg 6: 76-79.

7. Nehra D, Beynon J, Pye JK (1993) Spontaneous rupture of the esophagus (boerhaave's syndrome). Postgrad Med J 69: 214-216.
8. Khan AZ, Strauss D, Mason RC (2007) Boerhaave syndrome: diagnosis and surgical management. Surgeon 5: 39-44.

9. De Schipper JP, Pull ter Gunne AF, Oostvogel HJ, van Laarhoven CJ (2009) Spontaneous rupture of the oesophagus boerhaave syndrome in 2008. literature review and treatment algorithm. Dig Surg 26: 1-6.

10. Parkin GJ (1973) The radiology of perforated oesophagus. Clin Radiol 24: 324-332.

11. Petruzziello L, Tringali A, Riccioni ME, Mutignani M, Margaritora S, et al. (2003) Successful early treatment of Boerhaave's syndrome by endoscopic placement of a temporary self-expandable plastic stent without fluoroscopy. Gastrointest Endosc 58: 608-612.

12. Dumonceau JM, Deviere J, Cappello M, Gossum AV, Cremer M (1996) Endoscopic treatment of Boerhaave's syndrome. Gastrointest Endosc 44: 477-479.

13. Johnsson E, Lundell L, Liedman B (2005) Sealing of esophageal perforation or ruptures with expandable metallic stents: A prospective controlled study on treatment efficacy and limitations. Dis Esophagus 18: 262-266.

14. Sabanathan S, Eng J, Richardson J (1994) Surgical management of intrathoracic oesophageal rupture. Br J Surg 81: 863-865.

15. Matsuda A, Miyashita M, Sasajima K, Nomura T, Makino H, et al. (2006) Boerhaave syndrome treated conservatively following early endoscopic diagnosis: a case report. J. Nippon Med Sch 7: 341-345.

16. Sutcliffe RP, Forshaw MJ, Datta G, Rohatgi A, Strauss DC, et al. (2009) Surgical management of Boerhaave's syndrome in a tertiary oesophagogastric centre. Ann R Coll Surg Engl 91: 374-380.

17. Tanaka K, Tonouchi H, Konishi N, Ohmori Y, Mohri Y, et al. (2007) Cardiac tamponade as a postoperative complication of spontaneous esophageal rupture. Esophagus 4: 79-82. 